\title{
Flow Induced by an Oscillating Infinite Flat Plate in a Dusty Gas
}

\author{
J. T. C. LIU* \\ Daniel and Florence Guggenheim Jet Propulsion Center, Karmán Laboratory of Fluid Mechanics and Jet Propulsion, \\ California Institute of Technology, Pasadena, California
}

(Received 30 March 1966)

\begin{abstract}
The flow induced in an incompressible dusty gas by an infinite flat plate oscillating in its own plane is studied. The differential equation describing this problem is given; its form exhibits the relaxation from a frozen-diffusion process (corresponding to a clean gas) to an equilibrium-diffusion process (corresponding to a single heavier gas). The gas velocity profile, shear stress on the plate, and the particle velocity profile are obtained exactly and are discussed in terms of the parameters of the problem. The essential feature is the inhibition of viscous diffusion in the gas by the particle-gas velocity relaxation. Mechanical energy dissipation is discussed.
\end{abstract}

\section{INTRODUCTION}

$\mathrm{T}$ HE classical problem of an infinite oscillating plate was first considered by Stokes $^{2}$ in his paper on the effects of viscosity on the motion of pendulums. In the present paper, the corresponding problem is solved where the fluid is an incompressible viscous dusty gas. Here, the questions that naturally arise are the structure of the viscous diffusion layer, the fluid velocity, the shear stress at the plate due to particle-fluid-momentum interaction, and the particle "slip" relative to the fluid.

The viscous-diffusion layer thickness is expected to be decreased by the presence of the particle cloud. Part of the momentum generated by the plate motions that would otherwise diffuse into the interior of the mixture is now absorbed by the particle cloud. This then confines the periodic momentum produced at the wall to a region closer to the wall than in the absence of the particles. Since the particle cloud does not, in general, follow the fluid motion precisely, it is then expected that the phase lag of the fluid layers with respect to the plate is augmented by the presence of the particle cloud. Of course, the distortion of the fluid velocity profile due to particle inertia is expected to give rise to a relatively higher instantaneous shear stress at the plate.

In Sec. 2 the appropriate differential equation is given. In Sec. 3 , the solutions for the velocity profiles of both phases are obtained and the shear stress on the plate is also discussed. In Sec. 4 some discussion of the mechanical energy losses is presented.

\section{THE RELAXING DIFFUSION EQUATION}

The general conservation equations for the flow

* Present address: Center for Fluid Dynamics, Division of Engineering, Brown University, Providence, Rhode Island.

1 G. G. Stokes, Trans. Cambridge Phil. Soc. 9, 8 (1851); also in Mathematics and Physics Papers (Cambridge University Press, Cambridge, England, 1901), Vol. 3, p. 1. of a gas containing small solid particles are discussed and given by Marble. ${ }^{2}$ The form of the conservation equations in the case when the gas may be considered incompressible is given by Saffman. ${ }^{3}$

In the present problem, we make the assumption that the gas can be taken as incompressible. We assume the particles are spherical and are uniform in size. Following the above authors, the Stokes' form of the interaction force is assumed. The gas and particles are bounded by a flat plate, infinite in extent, at the $y=0$ plane as shown in Fig. 1 . We consider the motions of the plate to be in its own plane so that there is no displacement of gas in the $y$ direction due to plate motion. There is no expansion of the gas due to viscous dissipative heating under the incompressible assumption. In this case, the velocities of both the gas and the particle cloud in the $y$ direction are zero $\left(v=v_{p}=0\right)$. Furthermore, since the plate extends to infinity in the $\pm x$ directions, there are no changes in the $x$ direction of any property. The streamlines of the gas now coincide with that of the particle cloud and are parallel to the plate. In this situation, the density of the particle cloud $\rho_{p}$ is then a fixed distribution along the $y$ direction, but one which

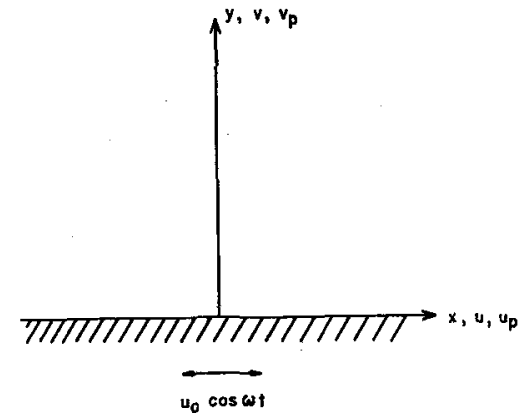

FIG. 1. Geometry of the problem. Schematic.

${ }^{2}$ F. E. Marble, in Proceeding of the Fifth AGARD Combustion and Propulsion Colloquium (Pergamon Press, Inc., New York, 1963), p. 175.

3 P. G. Saffiman, J. Fluid Mech. 13, 120 (1962). 
we take as constant throughout. The quantities $u$ and $u_{p}$ are, respectively, the gas and particle cloud velocities in the $x$ direction. They are now functions of $y$ and the time $t$ only.

The momentum equations thus obtained from Marble $^{2}$ or Saffman ${ }^{3}$ then appear in the form

$$
\begin{aligned}
\partial u / \partial t & =\nu\left(\partial^{2} u / \partial y^{2}\right)+(\kappa / \tau)\left(u_{p}-u\right), \\
\partial u_{p} / \partial t & =-\tau^{-1}\left(u_{p}-u\right),
\end{aligned}
$$

where $\kappa=\rho_{p} / \rho$, the particle to gas density ratio, is constant. $\nu=\mu / \rho$ is the kinematic viscosity of a clean gas. The particle relaxation time is $\tau$ and is $m / 6 \pi \mu r_{p}$, according to the Stokes' form of the interaction force between the two phases, where $r_{p}$ is the particle radius and $m$ is the mass of a single particle. The governing differential equations, (2.1) and (2.2), are rendered linear by the geometry of the problem and by the assumption that conditions when the gas can be considered incompressible are satisfied. Differentiating (2.1) with respect to $t$ once and using (2.2), we obtain the following "relaxing diffusion equation" for $u$ (or for $\left.u_{p}\right)^{4}$ :

$$
\frac{\tau}{1+\kappa} \frac{\partial}{\partial t}\left(\frac{\partial u}{\partial t}-\nu \frac{\partial^{2} u}{\partial y^{2}}\right)+\left(\frac{\partial u}{\partial t}-\bar{\nu} \frac{\partial^{2} u}{\partial y^{2}}\right)=0 .
$$

Here, $\bar{\nu}=\mu /\left(\rho+\rho_{p}\right)$ is the kinematic viscosity based on the density of the mixture and has the physical significance of being the "equilibrium diffusivity." On the other hand, $\nu$, being the kinematic viscosity of a clean gas, is the "frozen diffusivity." Both the gas and particle-cloud vorticity, $-\partial u / \partial y$ and $-\partial u_{p} / \partial y$, respectively, obey (2.3) as well.

The parameter indicating the extent of particlegas equilibration is the characteristic time of the plate motion $\omega^{-1}$ ( $\omega$ the frequency) divided by the particle velocity equilibration time $\tau$. It is then physically clear that there are two limiting regimes. When $1 /(\omega \tau) \rightarrow 0$, which is the frozen regime, the high-frequency viscous layer is so thin that it sees a negligible amount of particle cloud and hence renders the viscous diffusion process to be essentially that of a clean gas. In this situation, the first or the frozen-diffusion operator in (2.3) plays the dominant role. In the opposite limiting case, when $1 /(\omega \tau) \rightarrow \infty$, which is the equilibrium regime, the low-frequency viscous layer renders the gas and particle cloud to be in identical motion together so that the second or equilibrium-diffusion operator in (2.3) plays the dominant role. In both limiting cases, (2.3) reduces from third to second order. This

4. This equation appears in the same form as that governing elastic wave propagation in a visco-elastic solid for a threeparameter model (one elastic and two viscous elements) given by J. A. Morrison [Quart. Appl. Math. 14, 153 (1956)]. singular behavior, a usual situation characterizing nonequilibrium flows, is associated with the fact that in both cases the rate equation (2.2) becomes superfluous. The transition between the two diffusion processes for intermediary values of $1 /(\omega \tau)$ is then appropriately described by the full equation (2.3).

\section{PROPERTIES OF SOLUTION}

\subsection{Gas Velocity Profile}

Let us now consider the problem of an infinite flat plate oscillating parallel to its own plane with frequency $\omega$ and amplitude $u_{0}$. We restrict our attention to the $y>0$ plane. Our interest is in the induced motions of the two phases of the mixture. They are a result of the transmission of momentum produced at the plate into the interior of the mixture through the diffusive action of gas viscosity and the interaction between the gas and the particle cloud.

The boundary conditions for the gas velocity described in (2.3) are $u(0, t)=u_{0} \cos \omega t=R l\left(u_{0} e^{i \omega t}\right)$, and $u \rightarrow 0$ as $y \rightarrow \infty$. For periodic oscillations, $u(y, t)$ is proportional to $R l\left(e^{i \omega t}\right)$ and the solution is given by the form

$$
u(y, t)=R l\left(u_{0} e^{-\alpha y} e^{i \omega t}\right) .
$$

Then (2.3) gives

$$
\alpha^{2}=X_{1}+i Y_{1},
$$

where we have denoted the real and positive quantities $X_{1}$ and $Y_{1}$ by

$$
X_{1}=\frac{\omega}{\nu} \frac{\kappa(\omega \tau)}{1+(\omega \tau)^{2}}, \quad Y_{1}=\frac{\omega}{\nu} \frac{(1+\kappa)+(\omega \tau)^{2}}{1+(\omega \tau)^{2}} .
$$

The complex quantity $\alpha$ may be written as

$$
\alpha=X_{2}+i Y_{2}
$$

where $X_{2}$ and $Y_{2}$ are real. The solution of (3.4) is then obtained by the elementary means of equating real and imaginary parts of the same complex number,

$$
X_{1}=X_{2}^{2}-Y_{2}^{2}, \quad Y_{1}=2 X_{2} Y_{2} .
$$

Hence,

$$
\begin{aligned}
& X_{2}= \pm\left[X_{1} \pm\left(X_{1}^{2}+Y_{1}^{2}\right)^{\frac{1}{2}}\right]^{\frac{1}{2}} / \sqrt{2} \\
& Y_{2}= \pm\left[-X_{1} \pm\left(X_{1}^{2}+Y_{1}^{2}\right)^{\frac{1}{2}}\right]^{\frac{1}{2}} / \sqrt{2} .
\end{aligned}
$$

Since $\left(X_{1}^{2}+Y_{1}^{2}\right)^{\frac{1}{2}}>X_{1}$ and both $X_{2}, Y_{2}$ are real, the positive sign is chosen under the square root. For the sign outside of the square root, we choose the positive one for $X_{2}$ in the $y>0$ plane, since this gives the solution which vanishes as $y \rightarrow \infty$. Since $Y_{1}=2 X_{2} Y_{2}>0$, then the sign outside the square root for $Y_{2}$ must be chosen to be the same 
as that for $X_{2}$, namely, the positive one for the $y>0$ plane. Thus, finally,

$$
\begin{aligned}
& \left(\begin{array}{l}
X_{2} \\
Y_{2}
\end{array}\right)=\left(\frac{\omega}{2 \nu}\right)^{\frac{1}{2}} \\
& \cdot\left[\left(\begin{array}{l}
+1 \\
-1
\end{array}\right) \frac{\kappa(\omega \tau)}{1+(\omega \tau)^{2}}+\left(\frac{(1+\kappa)^{2}+(\omega \tau)^{2}}{1+(\omega \tau)^{2}}\right)^{\frac{1}{2}}\right]^{\frac{1}{2}} .
\end{aligned}
$$

The solution for the gas velocity profile is then

$$
u=u_{0} e^{-x_{s} \nu} \cos \left(\omega t-Y_{2} y\right) .
$$

As in the classical problem of Stokes, ${ }^{1}$ this result represents a transverse wave, one which propagates into the interior of the particle-gas mixture from the plate in a direction perpendicular to the plate motion. The wave velocity in this case is $\omega / Y_{2}$, and the wavelength is $2 \pi / Y_{2}$. The solution also exhibits the boundary-layer property, that is, the motion induced by the plate becomes insignificant when $X_{2} y$ reaches some value. $X_{2}^{-1}$ is the $e$-folding distance, which is of the order of the boundary-layer thickness $\delta$,

$$
\delta \sim\left(\frac{2 \nu}{\omega}\right)^{\frac{1}{2}}\left[\frac{\kappa(\omega \tau)}{1+(\omega \tau)^{2}}+\left(\frac{(1+\kappa)^{2}+(\omega \tau)^{2}}{1+(\omega \tau)^{2}}\right)^{\frac{1}{2}}\right]^{-\frac{1}{2}} .
$$

When the particle-gas density ratio $\kappa$ vanishes, both $X_{2}$ and $Y_{2}$ become $(\omega / 2 \nu)^{\frac{1}{2}}$ and the classical Stokes solution is recovered,

$$
u=u_{0} e^{-(\omega / 2 v)^{\natural} v} \cos \left[\omega t-(\omega / 2 \nu)^{\frac{1}{2}} y\right],
$$

with the boundary-layer thickness $\delta \sim(2 v / \omega)^{\frac{1}{2}}$.

Although the exact solution, given by (3.7) and (3.8), is in a form convenient for numerical calculation for any value of the equilibration parameter $1 /(\omega \tau)$, it is instructive to study the near limiting cases. When $1 /(\omega \tau) \gg 1$, which corresponds to the near-equilibrium regime, one can expand $X_{2}$ and $Y_{2}$ as follows:

$$
\begin{aligned}
& X_{2}=(\omega / 2 \bar{\nu})^{\frac{1}{2}}\left\{1+\kappa(\omega \tau) / 2(1+\kappa)+\vartheta\left[(\omega \tau)^{2}\right]\right\}, \\
& Y_{2}=(\omega / 2 \bar{\nu})^{\frac{1}{2}}\left\{1-\kappa(\omega \tau) / 2(1+\kappa)+\vartheta\left[(\omega \tau)^{2}\right]\right\} .
\end{aligned}
$$

In this case, it is found that the gas velocity profile can be written in the form

$$
\begin{aligned}
u / u_{0} & =u^{\prime}(\bar{\eta}, \bar{\xi}) \\
& =u_{\varepsilon_{0}}^{\prime}(\bar{\eta}, \bar{\xi})+(\omega \tau) u_{\epsilon_{1}}^{\prime}(\bar{\eta}, \bar{\xi})+\vartheta\left[(\omega \tau)^{2}\right] .
\end{aligned}
$$

Here, $\bar{\eta}=y(\omega / 2 \bar{\nu})^{\frac{3}{2}}$ is the similarity variable corresponding to the equilibrium kinematic viscosity $\bar{\nu}$; $\bar{\xi}$ is the oscillatory variable and is $(\omega t-\bar{\eta})$. The zeroth-order function is simply the equilibrium Stokes solution when the particle cloud and gas are moving together as a single heavier gas of density $(1+\kappa) \rho$

$$
u_{e}^{\prime}(\bar{\eta}, \bar{\xi})=e^{-\bar{\eta}} \cos \bar{\xi} .
$$

The first-order function is

$u_{e_{\mathrm{i}}}^{\prime}(\bar{\eta}, \bar{\xi})=-\left[\kappa \bar{\eta} e^{-\bar{\eta}} / 2(1+\kappa)\right](\sin \bar{\xi}+\cos \bar{\xi})$.

It is noticed that $(1+\kappa) u_{e_{1}}^{\prime} / \kappa$ is a universal function of $\bar{\eta}$ for the amplitude [and of $\bar{\xi}=(\omega t-\bar{\eta})$ for the periodic part] and is independent of $k$. This dependence is somewhat similar to the first-order function for the near-equilibrium limit in the nonlinear, steady, laminar boundary-layer problem discussed by Marble. ${ }^{2}$ The boundary-layer thickness approaches the equilibrium value in the manner

$$
\delta \sim(2 \bar{\nu} / \omega)^{\frac{t}{2}}[1-\kappa(\omega \tau) / 2(1+\kappa)] .
$$

When $1 /(\omega \tau) \ll 1$, which is the near-frozen regime, expansion of $X_{2}$ and $Y_{2}$ gives

$$
\begin{aligned}
& X_{2}=(\omega / 2 \nu)^{\frac{1}{2}}\left\{1+\kappa / 2(\omega \tau)+\vartheta\left[1 /(\omega \tau)^{2}\right]\right\}, \\
& Y_{2}=(\omega / 2 \nu)^{\frac{1}{2}}\left\{1-\kappa / 2(\omega \tau)+\vartheta\left[1 /(\omega \tau)^{2}\right]\right\} .
\end{aligned}
$$

In this case, one expects the gas velocity can be written as

$$
\begin{aligned}
u / u_{0} & =u^{\prime}(\eta, \xi) \\
& =u_{f_{0}}^{\prime}(\eta, \xi)+[1 /(\omega \tau)] u_{f_{1}}^{\prime}(\eta, \xi)+\vartheta\left[1 /(\omega \tau)^{2}\right],
\end{aligned}
$$

where $\eta=y(\omega / 2 \nu)^{\frac{1}{2}}$ is the similarity variable corresponding to the clean-gas kinematic viscosity $\nu$, $\xi=(\omega t-\eta)$. The zeroth-order function is simply the frozen Stokes solution

$$
u_{f_{0}}^{\prime}(\eta, \xi)=e^{-\eta} \cos \xi .
$$

The first-order function is

$$
u_{f_{1}}^{\prime}(\eta, \xi)=-\frac{1}{2} \kappa \eta e^{-\eta}(\sin \xi+\cos \xi) .
$$

$u_{f_{1}}^{\prime}(\eta, \xi) / \kappa$ is a universal function of $\eta$ for the amplitude and of $\xi=\omega t-\eta$ for the periodic part and is independent of $\kappa$. The boundary-layer thickness begins to break away from the frozen value in the manner

$$
\delta \sim(2 \nu / \omega)^{\frac{1}{2}}[1-\kappa / 2(\omega \tau)] .
$$

One notices here that the zeroth-order function for the near-frozen regime is identical to the clean-gas solution obtained by setting $\kappa$ identically to zero. In this case, even though the particle cloud remains frozen and the relative lag with respect to the gas motion is largest, the high-frequency, thin, viscous layer hardly notices the presence of the particle cloud. Thus, in the absence of effective interaction in this situation, the zeroth-order frozen limit behaves as if in the absence of the particle cloud. This situation is similar to, for instance, the region close to the leading edge of a steady laminar boundary layer on a flat plate.

Figure 2 shows the relaxation of the dimensionless wavelength parameter $\left[Y_{2}(2 \nu \tau)^{\frac{1}{2}}\right]^{-1}$ and the di- 


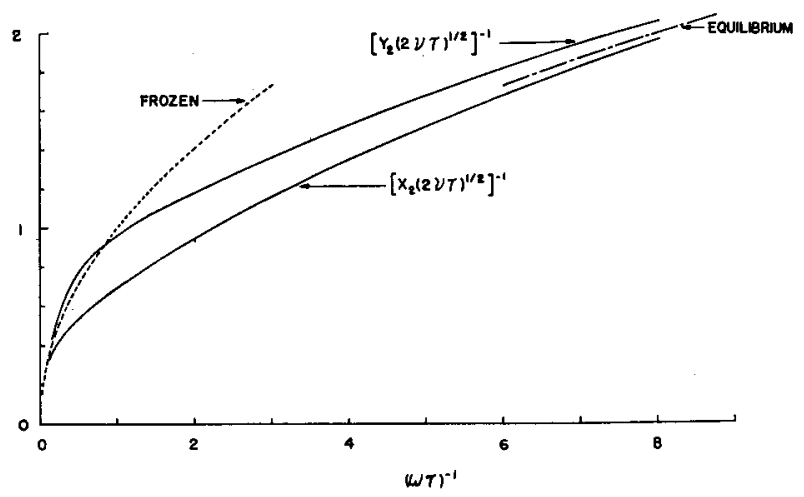

FIG. 2. Dimensionless wavelength parameter $\left[Y_{2}(2 \nu \tau)^{\frac{1}{2}}\right]^{-1}$ and boundary-layer-thickness parameter $\left[X_{2}(2 \nu \tau)^{\frac{1}{2}}\right]^{-1},(\kappa=1)$.

mensionless boundary-layer-thickness parameter $\left[X_{2}(2 \nu \tau)^{\frac{1}{2}}\right]^{-1}$ for $\kappa=1$. They are equal at both ends of the relaxation parameter $1 /(\omega \tau)$, as provided by the classical Stokes solution.

\subsection{Shear Stress at the Wall}

The frictional force exerted by the gas on a unit area of the plate surface is

$$
\tau_{w}=\mu(\partial u / \partial y)_{y=0} .
$$

Substituting (3.8) into (3.20) and forming the coefficient of skin friction, we obtain

$$
\begin{aligned}
c_{f} / 2 & =\tau_{w} / \rho u_{0}^{2} \\
& =\left(\nu / u_{0}\right)\left(X_{2}^{2}+Y_{2}^{2}\right)^{\frac{1}{2}} \cos (\omega t+\theta),
\end{aligned}
$$

where $\theta=\tan ^{-1}\left(Y_{2} / X_{2}\right)$. In the absence of the particle cloud $\kappa=0$, then $\theta$ becomes $\frac{1}{4} \pi$ and $\left(\nu / u_{0}\right)\left(X_{2}^{2}+Y_{2}^{2}\right)^{\frac{1}{2}}$ becomes $\left(\omega \nu / u_{0}^{2}\right)^{\frac{1}{2}}$, and

$$
\frac{1}{2} c_{f}=\left(\omega \nu / u_{0}^{2}\right)^{\frac{1}{2}} \cos \left(\omega t+\frac{1}{4} \pi\right) .
$$

Equation (3.22) is the classical expression for the Stokes friction coefficient for the oscillating plate, where the maximum of the shear stress at the wall occurs at intervals of $\frac{1}{8}$ of a period behind the mean position of the plate.

For the near equilibrium limit $1 /(\omega \tau) \gg 1$, then $\theta \cong \frac{1}{4} \pi-\kappa(\omega \tau) / 2(1+\kappa)$ and the additional lag $-\kappa(\omega \tau) / 2(1+\kappa)$ due to the inertia of the particle cloud now renders the maximum of the shear stress at the wall to take place at intervals of $\left[\frac{1}{8}-\kappa(\omega \tau) / 4 \pi(1+\kappa)\right]$ of a period behind the mean position of the plate. Expanding (3.21) for this situation, we obtain

$$
\begin{aligned}
& \frac{1}{2} c_{f}=(1+\kappa)\left(\omega \bar{\nu} / u_{0}^{2}\right)^{\frac{1}{2}}\left\{\cos \left(\omega t+\frac{1}{4} \pi\right)\right. \\
& \left.+[\kappa(\omega \tau) / 2(1+\kappa)] \sin \left(\omega t+\frac{1}{4} \pi\right)+\vartheta\left[(\omega \tau)^{2}\right]\right\} .
\end{aligned}
$$

The coefficient $(1+\kappa)$ in (3.23) is present because we normalized $\tau_{w}$ by $\rho u_{0}^{2}$ instead of by $(1+\kappa) \rho u_{0}^{2}$ for the limiting equilibrium case. The first term in

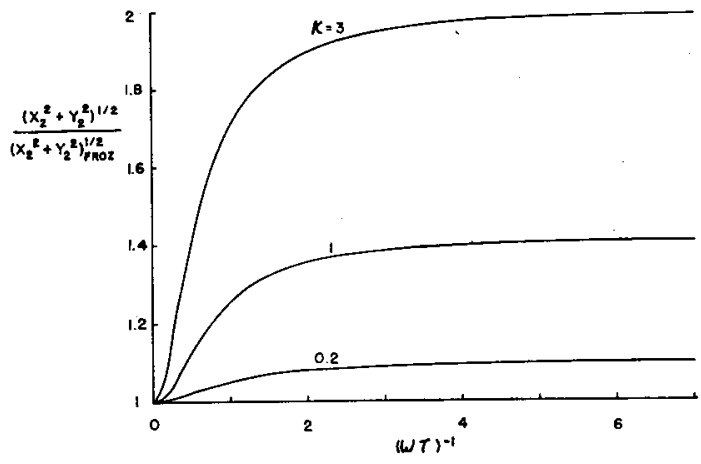

FIG. 3. Dimensionless skin-friction amplitude factor.

(3.23) corresponds to the equilibrium Stokes skin friction coefficient.

In the near frozen limit, $1 /(\omega \tau) \ll 1$, then $\theta \cong$ $\frac{1}{4} \pi-\kappa / 2(\omega \tau)$ and the maximum of the shear stress at the wall takes place at $\left[\frac{1}{8}-\kappa / 4 \pi(\omega \tau)\right]$ of a period behind the mean position of the plate. The skin friction coefficient then appears in the form

$$
\begin{aligned}
& \frac{1}{2} c_{f}=\left(\omega \nu / u_{0}^{2}\right)^{\frac{2}{2}}\left\{\cos \left(\omega t+\frac{1}{4} \pi\right)\right. \\
& \left.\quad+[\kappa / 2(\omega \tau)] \sin \left(\omega t+\frac{1}{4} \pi\right)+\vartheta\left[1 /(\omega \tau)^{2}\right]\right\},
\end{aligned}
$$

where the first term is the frozen Stokes' skin-friction coefficient.

The relaxation of the dimensionless skin-friction amplitude factor $\left(X_{2}^{2}+Y_{2}^{2}\right)^{\frac{1}{2}}\left(X_{2}^{2}+Y_{2}^{2}\right)_{\mathrm{froz}}^{-\frac{1}{2}}$, where the denominator is simply $(\omega / \nu)^{\frac{1}{2}}$, is shown in Fig. 3 . In the equilibrium limit, it simply becomes $(\nu / \bar{\nu})^{\frac{1}{2}}=$ $(1+\kappa)^{\frac{1}{2}}$. The relaxation of the skin-friction phase-lag angle $\theta$ is shown in Fig. 4 .

\subsection{Particle Velocity Profile}

The appropriate momentum equation (2.2) expresses the fact that the particle cloud is locally accelerated by the gas motion. The velocity profile of the particle cloud then takes the form

$$
u_{p}=u_{0} \cos \psi e^{-x_{2} y} \cos \left(\omega t-Y_{2} y-\psi\right) \text {, }
$$

where $\psi=\tan ^{-1}(\omega \tau)$. It is noted that the multiplicative amplitude factor $\cos \psi$ and the particle lag $\psi$ are the same for all layers of $y$. This is what is physically expected, since the particles are non-

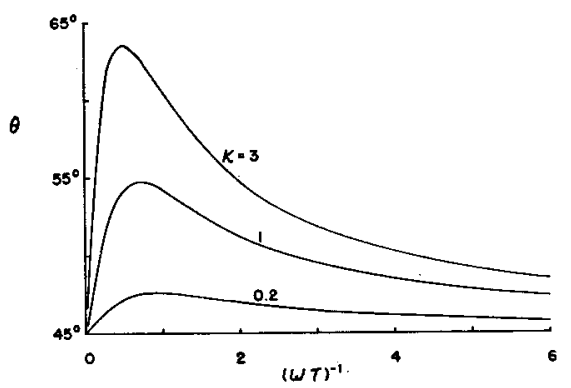

FIG. 4. Skin-friction phase-lag angle. 
interacting and respond only to the momentum exchange with the gas along each stream line. It is of interest to consider the particle-gas "slip" velocity, which is

$u-u_{p}=u_{0} \sin \psi e^{-X 2 y} \sin \left(\omega t-Y_{2} y-\psi\right)$.

The phase lag angle $\psi$ varies from $\frac{1}{2} \pi$ when $1 /(\omega \tau) \rightarrow 0$ to zero when $1 /(\omega \tau) \rightarrow \infty$.

In the near-equilibrium regime, the approximation for the slip velocity when $1 /(\omega \tau) \gg 1$ may be written in the form

$$
\left(u-u_{p}\right) / u_{0}=(\omega \tau) g_{e_{1}}(\bar{\eta}, \bar{\xi})+\vartheta\left[(\omega \tau)^{2}\right],
$$

where the first-order equilibrium slip function is given by

$$
g_{e_{1}}(\bar{\eta}, \bar{\xi})=e^{-\bar{\eta}} \sin \bar{\xi}
$$

Recalling that we previously defined $\bar{\eta}=y(\omega / 2 \bar{\nu})^{\frac{1}{2}}$ and $\bar{\xi}=(\omega t-\bar{\eta})$, corresponding to the equilibrium kinematic viscosity $\bar{\nu}$. Here, $g_{b_{x}}$ is a universal function of $(\bar{\eta}, \bar{\xi})$ and is independent of $\kappa$. We note here that the local acceleration of the zeroth-order equilibrium flow is given by

$$
\omega u_{0} e^{-\bar{\eta}} \sin \bar{\xi}
$$

Hence we recover from the exact solution the useful concept that the first-order slip velocity is proportional to the local acceleration of the zeroth-order flow in the near-equilibrium approximation. This is not surprising, since the particle of mass $m$ is subjected to an inertia force of order $m u_{0} \omega$ and this must be balanced by the force exerted on the particle by the gas of the order $m u_{s} / \tau$, where $u_{s}$ is the slip velocity. Hence, $u_{s} / u_{0} \approx(\omega \tau)$. Thus, when $1 /(\omega \tau) \gg 1$, the slip velocity is small compared to the amplitude of plate motion. It is interesting to note that this feature, which is recovered from expansions of the exact solution, essentially substantiates the approximation used by Marble $^{2}$ for the steady laminar boundary layer in the near-equilibrium limit.

In the opposite situation, when $1 /(\omega \tau) \ll 1$, it

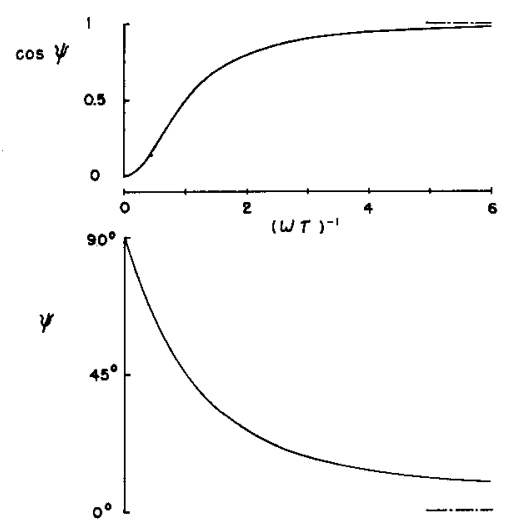
velocity amplitude factor $\cos \psi$ and phase-lag angle $\psi$ (independent of $\kappa$ ). is more convenient to write the particle velocity directly instead of the slip velocity

$$
u_{p} / u_{0}=[1 /(\omega \tau)] u_{p_{f_{1}}}^{\prime}(\eta, \xi)+\vartheta\left[1 /(\omega \tau)^{2}\right],
$$

where

$$
u_{p_{f_{1}}}^{\prime}(\eta, \xi)=e^{-\eta} \sin \xi \text {. }
$$

Again, $\eta=(\omega / 2 \nu)^{\frac{1}{2}} y$ and $\xi=\omega t-\eta$ are defined according to the clean gas or frozen kinematic viscosity $\nu$. The particle oscillations lag behind the fluid by $\frac{1}{2} \pi$ in the first approximation. The amplitude of particle motion is simply proportional to $1 /(\omega \tau)$, and the particle cloud is nearly standing still in this regime.

Both the particle velocity amplitude factor $\cos \psi$ and the phase lag angle $\psi$ are shown in Fig. 5.

\section{MECHANICAL ENERGY DISSIPATION}

Since the plate motion does a certain amount of work on the mixture as a whole (directly on the gas, indirectly on the particle cloud), part of this goes into increasing the overall kinetic energy of the mixture while the remainder is converted into heat through dissipation. There are two dissipative mechanisms here. They are the usual gas viscous dissipation and the work done due to particle-gas interaction. This may be simply demonstrated in the following.

Let us consider the overall kinetic energy of the mixture contained in a volume of height $y \rightarrow \infty$ and of unit area of plate surface

$$
E_{\mathrm{kin}}=\int_{0}^{\infty}\left(\frac{1}{2} \rho u^{2}+\frac{1}{2} \rho_{p} u_{p}^{2}\right) d y
$$

The rate of increase of $E_{\mathrm{kin}}$ can be written with the help of the momentum equations (2.1) and (2.2), which gives

$$
\begin{aligned}
& \frac{\partial E_{\text {kin }}}{\partial t}=-u(0, t) \tau_{w} \\
& -\left[\mu \int_{0}^{\infty}\left(\frac{\partial u}{\partial y}\right)^{2} d y+\left(\frac{\rho_{p}}{\tau}\right) \int_{0}^{\infty}\left(u_{p}-u\right)^{2} d y\right] .
\end{aligned}
$$

The first term on the right of (4.2) is the work done on the gas by the plate motion. The second term gives the rate at which the gas kinetic energy is dissipated through the gas viscosity, and the third term is the rate at which both the particle and gas kinetic energies are dissipated through particle-gas momentum interaction.

\section{ACKNOWLEDGMENTS}

The author is indebted to Professor Frank E. Marble for frequent and enlightening discussions.

This work was supported by the National Science Foundation under Grant 713. 